\title{
Functionalized Graphene Nanoplatelets from Ball Milling for Energy Applications
}

\author{
Xueliu Fan, ${ }^{1}$ Dong Wook Chang, ${ }^{2}$ Xuli Chen, ${ }^{1}$ Jong-Beom Baek, ${ }^{3}$ Liming Dai ${ }^{1}$ \\ 1 Department of Macromolecular Science and Engineering, Case Western Reserve University \\ 10900, Euclid Ave. Cleveland, OH 44106 USA \\ 2 Department of Industrial Chemistry, Pukyung National University \\ 100, Yongdang, Pusan 608-739, South Korea \\ 3 School of Energy and Chemical Engineering/Center for Dimension-Controllable Organic Frameworks, \\ Ulsan National Institute of Science and Technology (UNIST) \\ 50, UNIST, Ulsan 689-798, South Korea
}

\begin{abstract}
Having a large surface area, high mechanical strength, excellent electrical and thermal properties, graphene is attractive for a wide range of potential applications, including energy conversion and storage. To realize commercial reality of graphene-based energy devices, it is highly desirable to produce highquality graphene at a low cost and large scale. In this review, we will give an overview on large scale production of edge-selectively functionalized graphene nanoplatelets by mechanochemical ball-milling and their uses for energy conversion and storage.
\end{abstract}

\section{Introduction}

Owing to the continuous increasing global energy consumption, it has become more important than ever to develop efficient conversion and storage systems, such as solar cells, fuel cells, supercapacitors and lithium ion batteries. In this regard, innovative methods need to be developed for large-scale, low-cost production of materials for high-performance energy devices [1]. Because of its peculiar structure and unique properties, graphene, an atomic thick two-dimensional $\mathrm{sp}^{2}$ hybridized carbon network, has attracted considerable interest for various applications, including energy conversion and storage [2-6]. Functionalization of graphene can impart processability and further enhance its properties for efficient energy conversion and storage [7,8].

Along with the rapid development of carbon nanoscience and nanotechnology, several innovative methods have been reported recently for production of graphene, including mechanical exfoliation [9], epitaxial growth on $\mathrm{SiC}$ [10], chemical vapor deposition (CVD) [11], chemical exfoliation of graphite [12], sonication/intercalation [13], and ball milling [14]. Having involved complicated fabrication equipment and process, however, the catalytic growth of graphene by CVD is expensive for a large scale production. . Chemical exfoliation of graphite, involving acid oxidation of graphite, often leads to a significant damage of the graphene basal plane. Although subsequent reduction of the graphene oxide can partially recover the damaged basal plane structure, graphene materials produced by the solution method 
usually have lower conductivities and more defects than those of graphene generated by the ball milling method. To overcome these limitations, we have recently developed an innovative ball milling technology for low-cost scalable production of edge-functionalized graphene. In this article, we present an overview on the production of edge-functionalized graphene materials by ball milling and their applications in energy conversion and storage devices.

\section{Functionalized graphene nanoplatelets by ball milling}

Ball milling is a simple but efficient approach for producing edge-functionalized graphene nanoplatelets. In a typical experiment, graphite powders are mixed with chemicals containing heteroatoms (e.g., dry ice [14], sulfur trioxide [15], melamine [16,17], polystyrene [18]) in a sealed jar, followed by high speed ball milling. The strong shear forces generated between high-speed rotating balls caused the mechanochemical cracking of the graphitic C-C bonds, leading to spontaneous incorporation of functional groups and/or heteroatoms at the broken edges of graphitic frameworks as well as the subsequent exfoliation of graphene nanoplatelets. By dry ball milling graphite powder in the presence of appropriate chemical(s) (gas, liquid and/or solid phases), various heteroatoms, such as nitrogen or halogen, can be introduced at the edge of graphene nanoplatelets [19, 20]. Furthermore, wet ball milling has also been used to synthesize functionalized graphene [18]. In this case, the balls were mixed with a solution/dispersion of graphite and heteroatom-containing chemicals. Typically, graphene materials produced by ball milling possess a smaller grain size $(<1 \mu \mathrm{m})$ than those synthesized by other methods like chemical exfoliation of graphite.

In general, both basal plane and edges of graphene can be functionalized by the chemical moieties [21]. The methods for functionalization of graphene can be classified into several different types, such as covalent $\mathrm{C}-\mathrm{C}$ coupling, noncovalent $\pi-\pi$ interaction, substitutional heteroatom doping of graphene, and hybridization with nanoparticles or other materials [22]. Chemical moieties can selectively functionalize the edges of graphene through the formation of covalent bonding. For example, edge-carboxylated graphite (ECG) can be produced by solid state ball milling graphite powder in the presence of dry ice (solid phase of carbon dioxide, $\mathrm{CO}_{2}$ ) (Figure 1a and 1b) [14]. Owing to the large repulsive forces between the edge-carboxylate groups, the resultant ECG can be efficiently exfoliated into a few-layer nanosheets in polar solvents (e.g. water), leading to its enhanced dispersibility in various solvents.

The edge carboxylate functional groups in the ECG can be readily confirmed by Fourier transform infrared (FT-IR) spectra with a strong $\mathrm{C}=\mathrm{O}$ stretching peak at $1718 \mathrm{~cm}^{-1}$, in conjunction with a unique sharp peak form $\mathrm{C}-\mathrm{O}$ stretching at $1250 \mathrm{~cm}^{-1}$ exclusively arising from $\mathrm{O}=\mathrm{C}-\mathrm{OH}$. Whereas, $\mathrm{GO}$ exhibits a 
broad $\mathrm{C}-\mathrm{O}$ stretching band due to the coexistence of $\mathrm{C}-\mathrm{OH}$ (hydroxyl), $\mathrm{C}-\mathrm{O}-\mathrm{C}$ (epoxy) and $\mathrm{O}=\mathrm{C}-\mathrm{OH}$ (carboxyl) groups on its basal plane and edge. The obtained ECG also exhibits a large surface area of $389.4 \mathrm{~m}^{2} / \mathrm{g}$. Upon thermal decarboxylation, ECG film shows an electrical conductivity as high as 1214 $\mathrm{S} / \mathrm{cm}$. In addition to carboxylic acid, various functional groups, such as amine $\left(-\mathrm{NH}_{2}\right)$ and sulfonic acid ($\left.\mathrm{SO}_{3} \mathrm{H}\right)$, can also be efficiently introduced at the edges of graphene nanoplatelets under similar ball-milling conditions.

Although the covalent functionalization of graphitic basal plane can be regarded as a powerful tool to produce functionalized graphene nanoplatelets, it can significantly destroy the $\pi-\pi$ conjugated structure. On the contrary, the non-covalent functionalization of graphene based on van der Waals forces or $\pi-\pi$ stacking of aromatic molecules can produce physically functionalized graphene, in which the unique graphitic natures can be largely preserved. Interestingly, the polystyrene (PS) functionalized graphene nanoplatelets have been successfully prepared by simply ball milling graphite in the presence of a PS solution (Figure 1c and 1d) [18]. In addition, the PS chains efficiently stacked onto the basal plane of graphene nanoplatelets through strong $\pi-\pi$ interactions. The repulsive forces between functionalized PS chains can prevent the restacking of graphene nanosheets. The resultant PS-grafted graphene/PS nanocomposites show extraordinary electrical properties with a rather low percolation threshold of about $2.7 \mathrm{wt} \%$, due to enhanced compatibility and dispersibility of functionalized graphene into the PS matrix.

Chemical doping of graphene with heteroatoms (e.g. nitrogen, boron) is one of the most feasible approaches to modulate its electronic properties. The doped graphene can show either n-type or p-type behavior with greatly altered electronic characteristics [21]. In this context, nitrogen-doped graphene nanoplatelets have attracted considerable attention due to their outstanding performance in various energy and electronic devices, including fuel cells, batteries, and field-effect transistors [23, 24]. Traditionally, nitrogen-doping of graphene often requires complicated processes and/or chemical reagents containing additional undesirable components in their structures. However, nitrogen $\left(\mathrm{N}_{2}\right)$, the most abundant constituent in air, can be considered as an ideal material for nitrogen-doping. Recently, the successful direct nitrogen fixation at the edges of graphene nanoplatelets by dry ball milling graphite in $\mathrm{N}_{2}$ atmosphere has been reported, though $\mathrm{N}_{2}$ is normally considered to be an inert diatomic gas due to its strong triple bond (Figure 1e) [19]. $\mathrm{N}_{2}$ can readily react with the active carbon species generated by the mechanochemical cracking of graphitic C-C bonds during the ball milling process. The direct edge nitrogen fixation of graphene nanoplatelets using $\mathrm{N}_{2}$ is driven by aromatization, which is a thermodynamically favorable process. The calculated binding energies of nitrogen onto the zigzag and armchair-shaped broken edges for -57.65 and $-45.20 \mathrm{kcal} / \mathrm{N}_{2}$ mol, respectively, clearly indicate a spontaneous reaction to from nitrogen-containing heterocycles at the broken edges of graphene 
nanoplatelets. The structure of nitrogen-doped graphene nanoplatelets by ball milling has been confirmed either by FT-IR, which shows strong aromatic C-N stretching peak at $1400 \mathrm{~cm}^{-1}$, or by high-resolution XPS spectra for $\mathrm{C} 1 \mathrm{~s}$ and $\mathrm{N}$ 1s that shows the typical aromatic $\mathrm{C}-\mathrm{N}$ in pyrazole (pyrrolic $\mathrm{N}$ ) and pyridazine rings (pyridinic $\mathrm{N}$ ) as dominant nitrogen configurations. The obtained nitrogen-doped graphene nanoplatelets demonstrate the superior electrocatalytic properties for energy conversion in fuel cells and dye-sensitized solar cells (DSSCs).

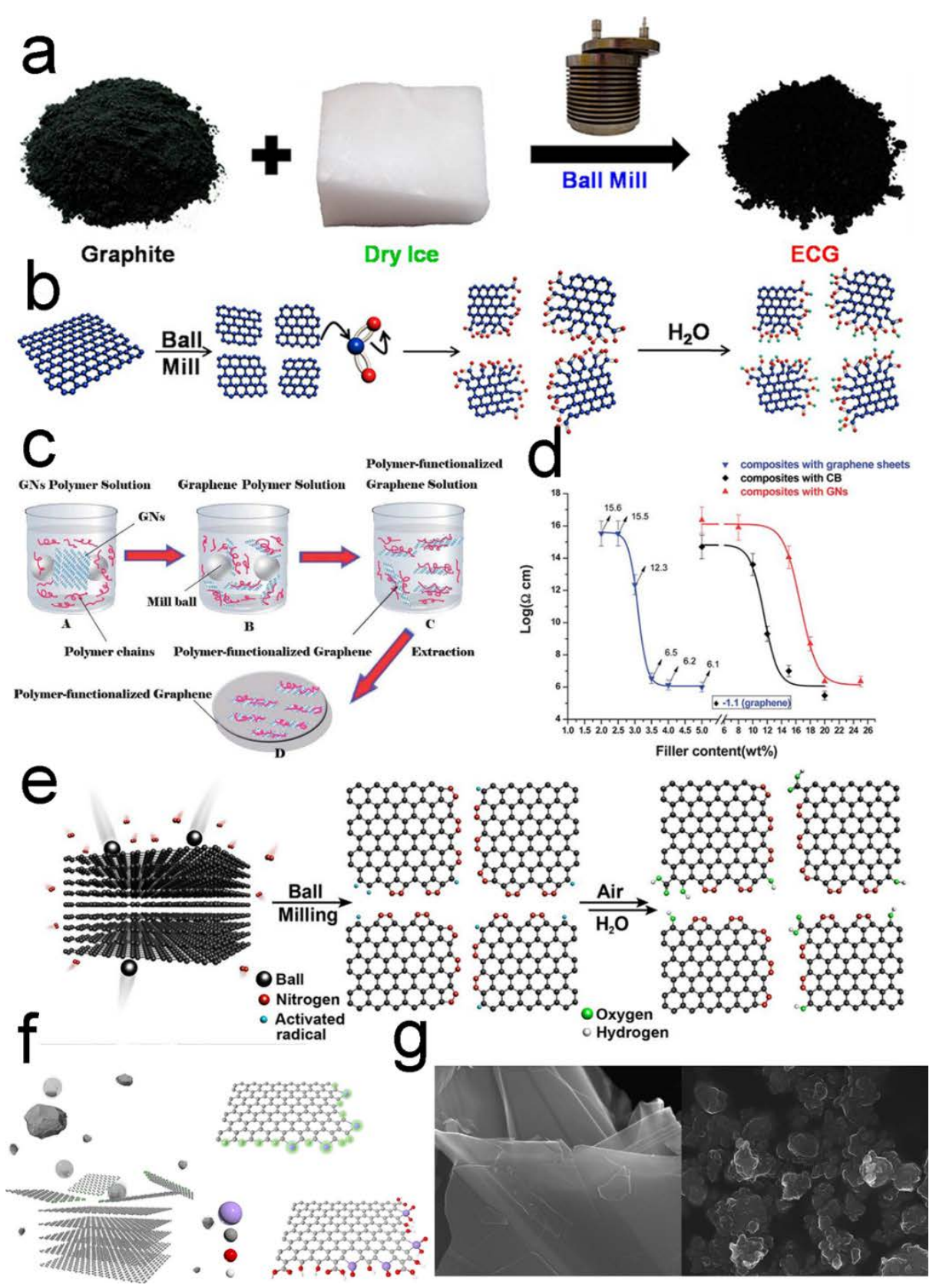

Figure 1. (a) Pristine graphite, dry ice (solid phase $\mathrm{CO}_{2}$ ); and edge-carboxylated graphite (ECG) prepared by ball milling for $48 \mathrm{~h}$; (b) a schematic illustration of the physical cracking and subsequent edge-carboxylation of graphite 
through ball milling, and the protonation by subsequent exposure to air moisture [14]. Reprinted from Ref. [14] with permission. Copyright 2012 National Academy of Sciences. (c) Schematic illustration of the preparation of polystyrene (PS) functionalized graphene nanoplatelets; (d) Curves of resistance versus filler content for composites with graphene, carbon black (CB) and PS-functionalized graphene [18]. Pristine Reprinted from Ref. [18] with permission. Copyright 2011 Royal Society of Chemistry. (e) A schematic illustration of physical cracking of graphite flake in a ball-mill crusher containing stainless steel balls agitated at $500 \mathrm{rpm}$ for $48 \mathrm{~h}$ in the presence of nitrogen and subsequent exposure to air moisture to produce N-graphene nanoplatelets [19] Reprinted from Ref. [19] with permission. Copyright 2013, Nature Publishing Group. (f) Schematic representation of mechanochemicall ball milling driven doping of antimony (Sb) to the edge of graphene nanoplatelets. SEM images of (g) before (Left Panel, $<150 \mu \mathrm{m}$ grain size) and after (Right Panel, $<1 \mu \mathrm{m}$ grain size) ball milling in the presence of solid state antimony [25]. Reprinted from Ref. [25] with permission. Copyright 2015, Nature Publishing Group.

Heteroatom doping of graphitic structures has been widely investigated for the development of functionalized graphene nanoplatelets. However, the heteroatom dopants have been limited to nonmetallic elements, such as nitrogen and boron. These materials do not satisfy a broad range of commercial demands for practical applications in terms of performance, cost and stability. Interestingly, the mechanochemical ball milling of graphite in the presence of solid antimony ( $\mathrm{Sb})$, which is one of melalloids, in a ball-mill crusher can efficiently produce edge-Sb doped graphene nanoplatelets (Figure $1 \mathrm{f}$ and $1 \mathrm{~g}$ ) [25]. The mechanochemically unzipped graphitic C-C bonds and activated Sb ore during ball milling process can induce the formation of $\mathrm{C}-\mathrm{Sb}$ bonds at the broken edges of graphene nanoplatelets. The doping levels of $\mathrm{Sb}$ in graphene structures can be reached up to $13.68 \mathrm{wt}$.\%. The structures of Sbdoped graphene nanoplatelets ( $\mathrm{SbGnP}$ ) have been confirmed by various analytical techniques, including atomic-resolution transmission electron microscopy (AR-TEM), X-ray photoelectron spectroscopy (XPS), scanning electron microscopy (SEM), thermogravimetric analysis (TGA), energy dispersive spectroscopy (EDS), Raman spectroscopy, and X-ray diffraction. Furthermore, the resultant SbGnPs exhibit superior electrocatalytic performance toward cathodic oxygen reduction reaction (ORR) with an enhanced tolerance against $\mathrm{CO}$ poisoning and methanol crossover, and profound long-term stability.

\section{Functionalized graphene nanoplatelets by ball milling for energy conversion}

\section{Functionalized graphene for dye-sensitized solar cells (DSSCs)}

DSSCs have been widely developed due to the low cost fabrication and high power conversion efficiency compared to the silicon-based solar cells [26]. A typical DSSC consists of a transparent conductive oxide, dye-adsorbed $\mathrm{TiO}_{2}$, electrolyte, and a counter electrode (CE). The counter electrode plays a crucial role in determining the device performance. An efficient counter electrode often requires electrode materials to have a high electrocatalytic activity for the redox couples and good electrical conductivity for charge transport [27]. Currently, expensive noble metals (e.g. Pt) have been widely used as the counter electrode in DSSCs. However, the noble metals are high cost and with a limited reserve in earth, and hence 
functionalized carbon-based nanomaterials have been intensively studied as low-cost, but efficient, alternatives [27,28].

Recently, the edged-carboxylated graphene nanoplatelets (ECGnPs) generated by ball milling process have been used as oxygen-rich metal-free counter electrodes [29]. Compared to the platinum counter electrodes, the ECGnPs based DSSC exhibited outstanding improvements in the electrochemical stability and charge-transfer properties for the $\mathrm{Co}(\mathrm{bpy})_{3}{ }^{2+/ 3+}$ redox couple due to the high charge polarization from the differences in the electronegativity between carbon $(\chi=2.55)$ and the oxygen $(\chi=3.50)$. Furthermore, the photovoltaic performance of ECGnPs based DSSC was enhanced with a fill factor of $74.4 \%$, shortcircuit current density of $14.07 \mathrm{~mA} / \mathrm{cm}^{2}$, and efficiency of $9.31 \%$, which were much higher than those of Pt, PEDOT:PSS, and reduced graphene oxide (RGO) based DSSCs (Figure 2). Similarly, N-doped graphene nanoplatelets can also be utilized as counter electrode in DSSCs with the extraordinary electrocatalytic properties as electrocatalysts for the $\mathrm{Co}(\mathrm{bpy})_{3}{ }^{2+/ 3+}$ redox reaction [30]. These results illustrated promising applications of the ball milled graphene nanoplatelets in highly efficient DSSCs.
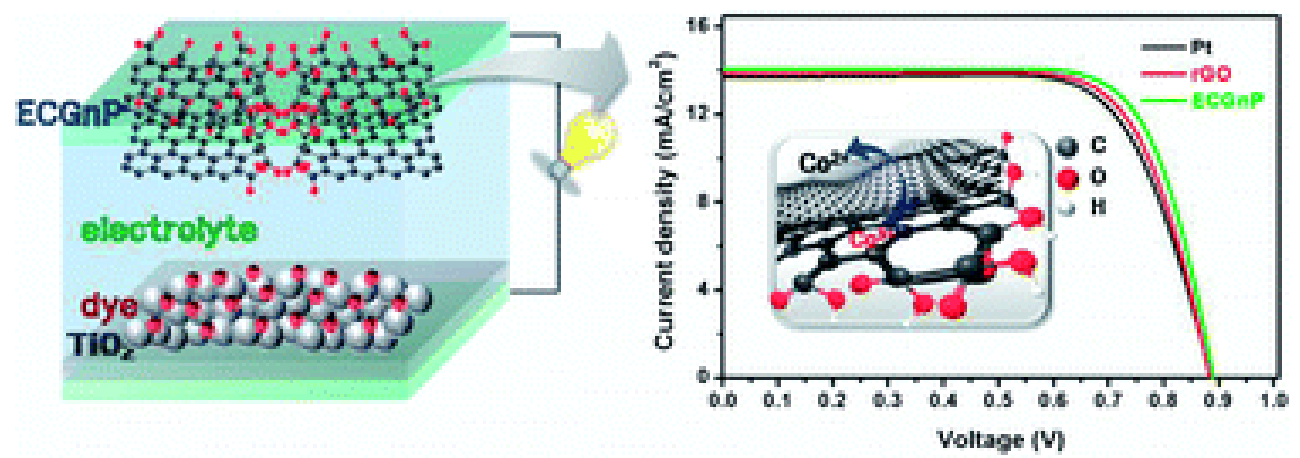

Figure 2. (a) The structure of DSSCs using ECGnPs as a counter electrode and (b) current-voltage characteristics of the DSSCs with the Pt, rGO and ECGnPs under one-sun illumination (AM1.5) [29]. Reprinted from Ref. [29] with permission. Copyright 2014, Royal Society of Chemistry.

\section{Functionalized graphene for fuel cells}

Fuel cell is one of the greenest energy devices that can directly convert chemical energy into electricity with a high efficiency but negligible emission, and thus it has attracted a great deal of interest [7]. Much efforts have been devoted to the development of oxygen reduction reaction (ORR) catalyst, which is the key component for the fuel cell performance [31]. Although platinum (Pt)-based catalysts have long been regarded as the most practical catalyst for fuel cells, their high cost, insufficient durability, and scarcity have prompted new search for non-precious, highly active, and stable ORR electrocatalysts for fuel cells [8,32]. In this context, carbon nanomaterials have been used as low-cost, metal-free ORR catalysts with extraordinary performance [31]. Particularly, the ball milling method has allowed large-scale production 
of functionalized graphene sheets at low cost as efficient metal-free ORR catalysts for fuel cell applications.
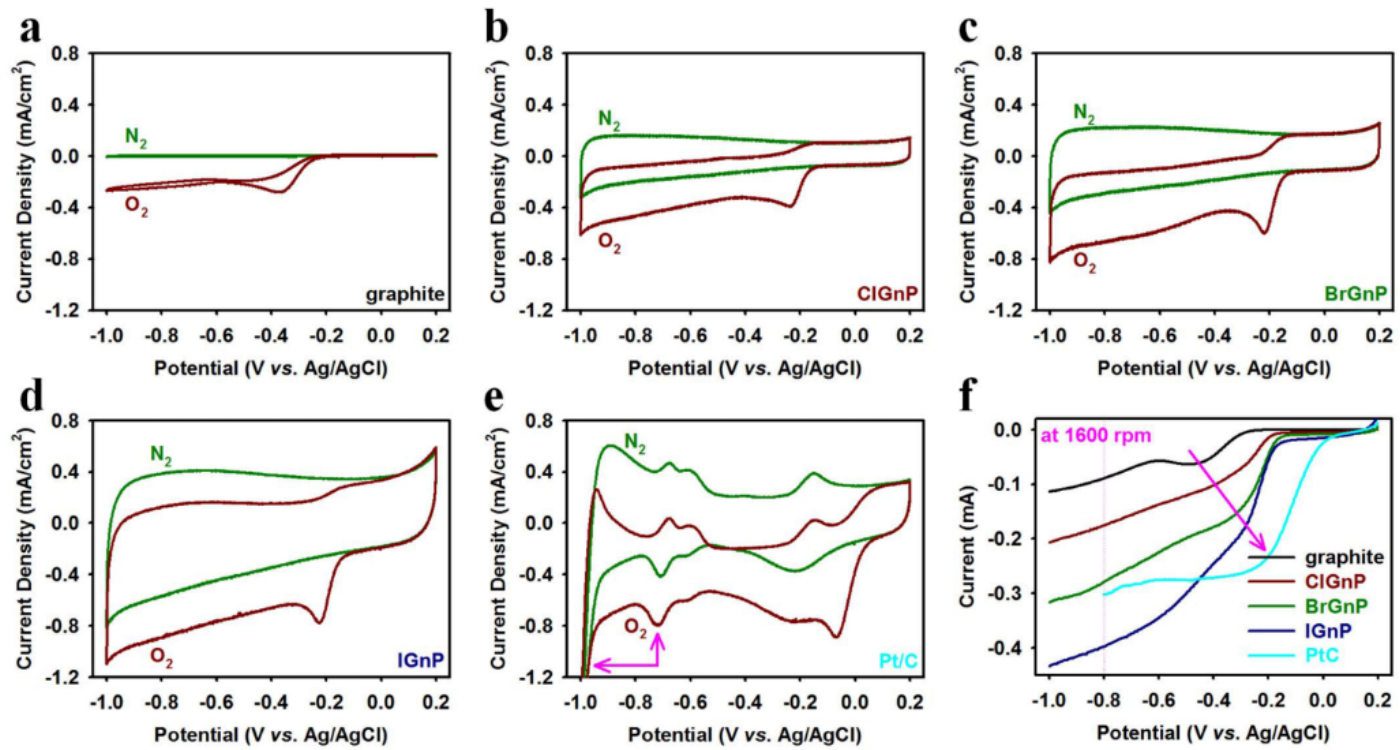

Figure 3. Cyclic voltammograms (CV) of (a) the pristine graphite; (b) $\mathrm{ClGnP}$; (c) $\mathrm{BrGnP}$; (d) IGnP; (e) $\mathrm{Pt} / \mathrm{C}$ on glassy carbon electrode in $\mathrm{N}_{2}$ or $\mathrm{O}_{2}$ saturated $0.1 \mathrm{M} \mathrm{KOH}$ aqueous solution at the scan rate of $10 \mathrm{mV} / \mathrm{s}$. (f) Linear sweep voltammograms (LSV) [20]. Reprinted from Ref. [20] with permission. Copyright 2013, Nature Publishing Group.

As mentioned above, the introduced heteroatoms in the graphene sheets can tailor the electronic structure for designed catalytic activities due to the difference in electronegativity between the heteroatom dopants and carbon atoms in the covalently doped graphene framework. Recent work on ball milling of graphite has reported to introduce $\mathrm{S}$ [15], $\mathrm{Cl}, \mathrm{Br}$, or I [20] as dopants for the edge-functionalized graphene nanoplatelets. Owing to the polarization of the adjacent carbon atoms by the heteroatoms in the graphite framework, the adsorption of $\mathrm{O}_{2}$ and the charge transfer have been improved, and hence enhancing the overall ORR activity. Therefore, all the resultant functionalized graphene materials thus produced showed remarkable ORR activities, comparable or better than commercial the Pt catalyst. Among all electrodes, the iodine doped nanoplatelets exhibited the highest capacitances of 127.6 and $139.5 \mathrm{~F} / \mathrm{g}$ in both $\mathrm{N}_{2}$ and $\mathrm{O}_{2}$ saturated electrolytes with a high cycle stability (Figure 3).

\section{Functionalized graphene nanoplatelets by ball milling for energy storage}

The energy storage devices, such as batteries and supercapacitors, are as important as the energy conversion devices. Therefore, numerous research efforts have also been devoted to improve the energy storage capability, power delivery capability, and cycling stability of batteries [33]. Recently, 
functionalized graphene materials from ball milling have been utilized for high-performance batteries and supercapacitors due to their high conductivity and the abundant active edge sites at their edges [34]. For instance, the edge-selectively hydrogenated $(\mathrm{H})$ and halogenated $(\mathrm{Cl}, \mathrm{Br}$, or I) graphene nanoplatelets by ball milling have been used as anode materials for lithium ion batteries (LIBs) [35]. The edge-iodinated graphene nanoplatelets based LIBs were found to deliver an initial charge capacity of $562.8 \mathrm{mAh} / \mathrm{g}$ at 0.5 $\mathrm{C}$ in a voltage range of $0.02-3.0 \mathrm{~V}$ (Figure 4a). Furthermore, the obtained LIBs exhibited a good cycling stability (charge capacity retention of $81.4 \%$ after 500 cycles), along with a good long-term life (high reversible capacity of $464.1 \mathrm{mAh} / \mathrm{g}$ after 1 month storage) (Figure $4 \mathrm{~b}$ ). Similarly, edge-fluorinated graphene nanoplatelets from ball milling have also been used as anode materials for LIBs, which exhibited a charge capacity of $650.3 \mathrm{mAh} / \mathrm{g}$ at $0.5 \mathrm{C}$ with charge retention of $76.6 \%$ after 500 cycles [36]. These results demonstrate that the edge-functionalized graphene nanoplatelets produced by the ball milling process are promising candidates as anode materials for high performance LIBs.
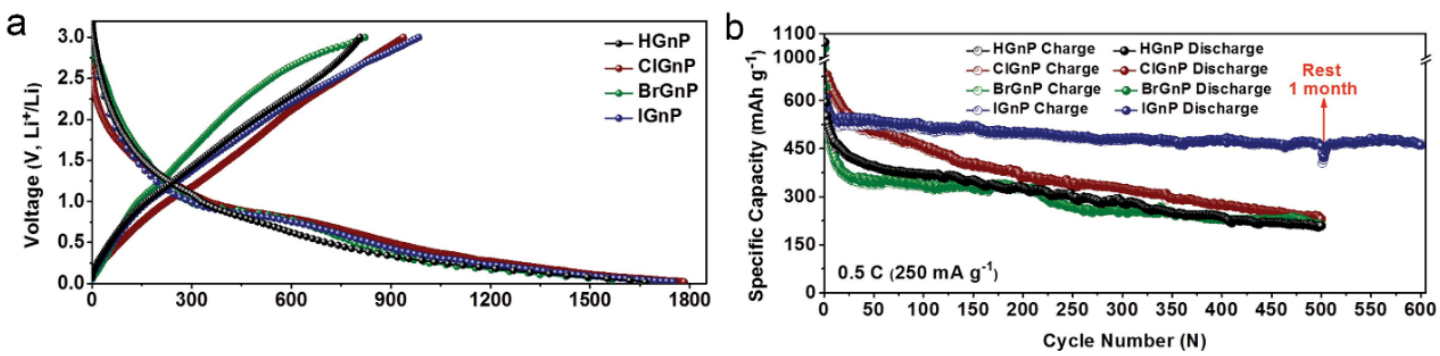

Figure 4. (a) Initial discharge-charge curves at $0.1 \mathrm{C}$; (b) cycling performance of H-graphene nanoplatelets and Xgraphene nanoplatelets $(\mathrm{X}=\mathrm{Cl}, \mathrm{Br}$ or I) at $0.5 \mathrm{C}$ in the voltage range of 0.02-3.0 V [35]. Reprinted from Ref. [35] with permission. Copyright 2014, John Wiley \& Sons, Inc.

In the past few years, sulfur-functionalized graphene has attracted considerable attention as the cathode material for lithium sulfur batteries (LSBs) [37,38]. High-performance LSBs have been constructed from edge-sulfur functionalized graphene nanoplatelets from ball milling. The edge-sulfur doped graphene nanoplatelets were found to deliver a high initial reversible capacity of $1265.3 \mathrm{mAh} / \mathrm{g}$ at $0.1 \mathrm{C}$, a high reversible capacity of $966.1 \mathrm{mAh} / \mathrm{g}$ at $2 \mathrm{C}$, and a low capacity decay rate of $0.099 \%$ per cycle over 500 cycles [37].

\section{Concluding remarks}

Graphene with its unique structure and properties have been widely used as electrode materials in energy conversion and storage devices. Functionalization of graphene materials can tailor their electrical and chemical properties, and hence broadening their potential applications. As one of the most promising methods for edge-functionalization, mechanochemical ball milling has been demonstrated to produce high-quality edge-selectively functionalized graphene nanoplatelets in large scale and at a low-cost and 
eco-friendly manner, opening new vistas for functionalization of graphene for energy conversion and storage, such as solar cells, fuel cells, supercapacitors, and batteries. Although much progress has been achieved, there is still considerable room for further optimizing the ball milling process to tailor-made the structures (e.g. size, shape, layer number of GnPs) and properties (e.g. electrocatalytic, electronic, chemical) for various specific applications, including energy conversion and storage.

\section{Acknowledgements}

This work is supported by Creative Research Initiative (CRI), BK21 Plus programs through the National Research Foundation (NRF) of Korea, DAGSI, NSF (CMMI-1400274, CMMI-1266295, AIR-IIP1343270, DMR 1106160), and DOD-AFOSR-MURI (FA9550-12-1-0037).

\section{References}

[1]. Dai, L.M., Chang, D.W., Baek, J.B. \& Lu, W. Carbon Nanomaterials for Advanced Energy Conversion and Storage. Small 8, 1130-1166 (2012).

[2]. Geim, A.K. \& Novoselov, K.S. The Rise of Graphene. Nature Materials 6, 183-191 (2007).

[3]. Geim, A.K. Graphene: Status and Prospects. Science 324, 1530-1534 (2009).

[4]. Novoselov, K.S., Geim, A.K., Morozov, S.V., Jiang, D., Katsnelson, M.I., Grigorieva, I.V., Dubonos, S.V. \& Firsov, A.A. Two-Dimensional Gas of Massless Dirac Fermions in Graphene. Nature 438, 197-200 (2005).

[5]. Balandin, A.A., Ghosh, S., Bao, W.Z., Calizo, I., Teweldebrhan, D., Miao, F. \& Lau, C.N. Superior Thermal Conductivity of Single-Layer Graphene. Nano Letters 8, $902-907$ (2008).

[6]. Lee, C., Wei, X.D., Kysar, J.W. \& Hone, J. Measurement of the Elastic Properties and Intrinsic Strength of Monolayer Graphene. Science 321, 385-388 (2008).

[7]. *Dai, L.M. Functionalization of Graphene for Efficient Energy Conversion and Storage.

Accounts of Chemical Research 46, 31-42 (2013).

This article provides a compressive summary of the new ideas and strategies for the controlled functionalization of graphene for applications in efficient energy conversion and storage devices. [8]. Qu, L.T., Liu, Y., Baek, J.B. \& Dai, L.M. Nitrogen-Doped Graphene as Efficient Metal-Free Electrocatalyst for Oxygen Reduction in Fuel Cells. ACS Nano 4, 1321-1326 (2010).

[9]. Novoselov, K.S., Geim, A.K., Morozov, S.V., Jiang, D., Zhang, Y., Dubonos, S.V., Grigorieva, I.V. \& Firsov, A.A. Electric Field Effect in Atomically Thin Carbon Films. Science 306, 666-669 (2004). [10]. Berger, C., Song, Z.M., Li, T.B., Li, X.B., Ogbazghi, A.Y., Feng, R., Dai, Z.T., Marchenkov, A.N., Conrad, E.H., First, P.N., et al. Ultrathin Epitaxial Graphite: 2D Electron Gas Properties and a 
Route toward Graphene-Based Nanoelectronics. Journal of Physical Chemistry B 108, 19912-19916 (2004).

[11]. Reina, A., Jia, X.T., Ho, J., Nezich, D., Son, H.B., Bulovic, V., Dresselhaus, M.S. \& Kong, J. Large Area, Few-Layer Graphene Films on Arbitrary Substrates by Chemical Vapor Deposition. Nano Letters 9, 30-35 (2009).

[12]. Park, S. \& Ruoff, R.S. Chemical Methods for the Production of Graphenes. Nature Nanotechnology 4, 217-224 (2009).

[13]. Lotya, M., Hernandez, Y., King, P.J., Smith, R.J., Nicolosi, V., Karlsson, L.S., Blighe, F.M., De, S., Wang, Z.M., McGovern, I.T., et al. Liquid Phase Production of Graphene by Exfoliation of Graphite in Surfactant/Water Solutions. Journal of the American Chemical Society 131, 3611-3620 (2009).

[14]. Jeon, I.Y., Shin, Y.R., Sohn, G.J., Choi, H.J., Bae, S.Y., Mahmood, J., Jung, S.M., Seo, J.M., Kim, M.J., Chang, D.W., et al. Edge-Carboxylated Graphene Nanosheets via Ball Milling. Proceedings of the National Academy of Sciences 109, 5588-5593 (2012).

[15]. Jeon, I.Y., Choi, H.J., Jung, S.M., Seo, J.M., Kim, M.J., Dai, L.M. \& Baek, J.B. Large-Scale Production of Edge-Selectively Functionalized Graphene Nanoplatelets via Ball Milling and Their Use as Metal-Free Electrocatalysts for Oxygen Reduction Reaction. Journal of the American Chemical Society 135, 1386-1393 (2013).

[16]. León, V., Quintana, M., Herrero, M.A., Fierro, J.L., De La Hoz, A., Prato, M. \& Vázquez, E. Few-Layer Graphenes from Ball-Milling of Graphite with Melamine. Chemical Communications 47, 10936-10938 (2011).

[17]. Leon, V., Rodriguez, A.M., Prieto, P., Prato, M. \& Vazquez, E. Exfoliation of Graphite with Triazine Derivatives under Ball-Milling Conditions: Preparation of Few-Layer Graphene via Selective Noncovalent Interactions. ACS Nano 8, 563-571 (2014).

[18]. Wu, H., Zhao, W.F., Hu, H.W. \& Chen, G.H. One-Step in Situ Ball Milling Synthesis of Polymer-Functionalized Graphene Nanocomposites. Journal of Materials Chemistry 21, 8626-8632 (2011).

[19]. Jeon, I.Y., Choi, H.J., Ju, M.J., Choi, I.T., Lim, K.M., Ko, J.J., Kim, H.K., Kim, J.C., Lee, J.J., Shin, D.B., et al. Direct Nitrogen Fixation at the Edges of Graphene Nanoplatelets as Efficient Electrocatalysts for Energy Conversion. Scientific Reports 3 (2013).

[20]. *Jeon, I.Y., Choi, H.J., Choi, M., Seo, J.M., Jung, S.M., Kim, M.J., Zhang, S., Zhang, L.P., Xia, Z.H., Dai, L.M., et al. Facile, Scalable Synthesis of Edge-Halogenated Graphene Nanoplatelets as Efficient Metal-Free Eletrocatalysts for Oxygen Reduction Reaction. Scientific Reports 3 (2013). 
This article reports a simple but efficient preparation of a series of edge-selectively halogenated $(\mathrm{X}=\mathrm{Cl}$, $\mathrm{Br}$, I) graphene nanoplatelets $(\mathrm{XGnPs}=\mathrm{ClGnP}, \mathrm{BrGnP}, \mathrm{IGnP})$ by ball-milling graphite in the presence of $\mathrm{Cl}_{2}, \mathrm{Br}_{2}$ and $\mathrm{I}_{2}$, respectively.

[21]. Loh, K.P., Bao, Q.L., Ang, P.K. \& Yang, J.X. The Chemistry of Graphene. Journal of Materials Chemistry 20, 2277-2289 (2010).

[22]. Georgakilas, V., Otyepka, M., Bourlinos, A.B., Chandra, V., Kim, N.D., Kemp, K.C., Hobza, P., Zboril, R. \& Kim, K. S. Functionalization of Graphene: Covalent and Non-Covalent Approaches, Derivatives and Applications. Chemical Reviews 112, 6156-6214 (2012).

[23]. Dai, L.M., Xue, Y.H., Qu, L.T., Choi, H.J. \& Baek, J.B. Metal-Free Catalysts for Oxygen Reduction Reaction. Chemical Reviews 115, 4823-4892 (2015).

[24]. Wang, H., Maiyalagan, T. \& Wang, X. Review on Recent Progress in Nitrogen-Doped Graphene: Synthesis, Characterization, and Its Potential Applications. ACS Catalysis 2, 781-794 (2012).

[25]. *Jeon, I.-Y., Choi, M., Choi, H.J., Jung, S.M., Kim, M.J., Seo, J.M., Bae, S.Y., Yoo, S.Y., Kim, G., Jeong, H.Y., et al. Antimony-Doped Graphene Nanoplatelets. Nature communications 6 (2015). In this article, doping semimetal antimony ( $\mathrm{Sb}$ ) at the edges of graphene nanoplatelets ( $\mathrm{GnPs}$ ) has been realized in the ball milling process, and the Sb-doped GnPs were demonstrated to display zero loss of electrocatalytic activity for oxygen reduction reaction even after 100,000 cycles. [26]. Oregan, B. \& Gratzel, M. A Low-Cost, High-Efficiency Solar-Cell Based on Dye-Sensitized Colloidal $\mathrm{TiO}_{2}$ Films. $_{\text {. }}$ Nature 353, 737-740 (1991).

[27]. Roy-Mayhew, J.D., Bozym, D.J., Punckt, C. \& Aksay, I.A. Functionalized Graphene as a Catalytic Counter Electrode in Dye-Sensitized Solar Cells. ACS Nano 4, 6203-6211 (2010).

[28]. Lee, W.J., Ramasamy, E., Lee, D.Y. \& Song, J.S. Efficient Dye-Sensitized Solar Cells with Catalytic Multiwall Carbon Nanotube Counter Electrodes. ACS applied materials \& interfaces 1, 11451149 (2009).

[29]. **Ju, M.J., Jeon, I.Y., Lim, K.M., Kim, J.C., Choi, H.J., Choi, I.T., Eom, Y.K., Kwon, Y.J., Ko, J.J., Lee, J.J., et al. Edge-Carboxylated Graphene Nanoplatelets as Oxygen-Rich Metal-Free Cathodes for Organic Dye-Sensitized Solar Cells. Energy \& Environmental Science 7, 1044-1052 (2014).

This article reported the simple and efficient ball milling preparation of edge-carboxylated graphene nanoplatelets, which have been applied as oxygen-rich metal-free counter electrodes in the organic dyesensitized solar cells to exhibit a significant improvement in device performance compared to the platinum counter electrode.

[30]. Ju, M.J., Jeon, I.Y., Kim, J.C., Lim, K.M., Choi, H.J., Jung, S.M., Choi, I.T., Eom, Y.K., Kwon, Y.J., Ko, J.J., et al. Graphene Nanoplatelets Doped with N at its Edges as Metal-Free Cathodes for Organic Dye-Sensitized Solar Cells. Advanced Materials 26, 3055-3062 (2014). 
[31]. Yu, D.S., Nagelli, E., Du, F. \& Dai, L.M. Metal-Free Carbon Nanomaterials Become More Active than Metal Catalysts and Last Longer. Journal of Physical Chemistry Letters 1, 2165-2173 (2010).

[32]. Zhang, J., Sasaki, K., Sutter, E. \& Adzic, R.R. Stabilization of Platinum Oxygen-Reduction Electrocatalysts Using Gold Clusters. Science 315, 220-222 (2007).

[33]. Etacheri, V., Marom, R., Elazari, R., Salitra, G. \& Aurbach, D. Challenges in the Development of Advanced Li-Ion Batteries: A Review. Energy \& Environmental Science 4, 3243-3262 (2011).

[34]. Chen, T. \& Dai, L.M. Carbon Nanomaterials for High-Performance Supercapacitors. Materials Today 16, 272-280 (2013).

[35]. **Xu, J.T., Jeon, I.Y., Seo, J.M., Dou, S.X., Dai, L.M. \& Baek, J.B. Edge-Selectively Halogenated Graphene Nanoplatelets (XGnPs, $\mathrm{X}=\mathrm{Cl}$, Br, or I) Prepared by Ball-Milling and Used as Anode Materials for Lithium-Ion Batteries. Advanced Materials 26, 7317-7323 (2014).

This article reports the ball milling preparation of edge-selectively halogenated graphene materials, which achieved excellent electrochemical performances as anode in the lithium-ion batteries with good cycling stability.

[36]. Jeon, I.Y., Ju, M.J., Xu, J.T., Choi, H.J., Seo, J.M., Kim, M.J., Choi, I.T., Kim, H.M., Kim, J.C., Lee, J.J., et al. Edge-Fluorinated Graphene Nanoplatelets as High Performance Electrodes for DyeSensitized Solar Cells and Lithium Ion Batteries. Advanced Functional Materials 25, 1170-1179 (2015).

[37]. Xu, J.T., Shui, J.L., Wang, J.L., Wang, M., Liu, H.K., Dou, S.X., Jeon, I.Y., Seo, J.M., Baek, J.B. \& Dai, L.M. Sulfur-Graphene Nanostructured Cathodes via Ball-Milling for High-Performance LithiumSulfur Batteries. ACS Nano 8, 10920-10930 (2014).

[38]. Cao, Y.L., Li, X.L, Aksay, I.A., Lemmon, J., Nie, Z.M., Yang, Z.G. \& Liu, J.

Sandwich-Type Functionalized Graphene Sheet-Sulfur Nanocomposite for Rechargeable Lithium Batteries. Physical Chemistry Chemical Physics 13, 7660-7665 (2011). 